\title{
Optimal deployment of energy storage systems in a DC-electrified railway system
}

\author{
A. J. López-López, R. R. Pecharromán, J. A. García-Matos, \\ A. Fernández-Cardador \& A. P. Cucala \\ Institute for Research in Technology, ICAI School of Engineering, \\ Comillas Pontifical University, Madrid, Spain
}

\begin{abstract}
Energy Storage Systems (ESSs) represent one of the main vectors for improving railway electrical systems. Former low reliability rates and life-time are being dramatically improved in recent times due to the intensive research on battery and capacitor technologies. However, little research has been conducted on the optimization of railway system infrastructure by incorporating ESSs. This paper presents a method for designing the best possible deployment of ESSs in a DCelectrified mass rapid transport system line by means of optimization, selecting number and location of ESSs. A detailed model of system load flow and energy storage devices for a given traffic scenario in a general DC railway system has been carried out, resulting in a MINLP optimisation model that minimises total energy consumption. Then, a linearization of load flow equations has been performed in order to get a fast-approximate solution of the system by converting the MINLP problem into a MIP one. This optimization method has been applied to a realistic railway system, yielding reasonable location and number of ESSs in the system. The error due to the approximation has been evaluated by comparing the results of the linear load flow against those of the non-linear load flow. The method has demonstrated to be a powerful tool to decide how many and where to place ESSs in a DC-electrified railway system. Keywords: optimisation, energy storage systems, mass rapid transport systems.
\end{abstract}

Notation

\begin{tabular}{ll}
$p E \max$ & Maximum energy in the ESS. \\
\hline$p G i j$ & Conductance linking nodes $\mathrm{i}$ and $\mathrm{j}$ \\
\hline$p M$ & Huge number.
\end{tabular}




\begin{tabular}{ll}
$p P C \max$ & Maximum admissible charging power rate. \\
\hline$p P$ dem & Power demanded by a train in a given instant. \\
\hline$p P g e n$ & Power regenerated by a train in a given instant. \\
\hline$p V 1$ & Voltage in the slack node. \\
\hline$p V$ charge & Voltage threshold to start charging. \\
\hline$p V$ dis & Voltage threshold to start discharging. \\
\hline$p V$ max & Maximum admissible voltage in the line. \\
\hline$p E f f$ & ESS efficiency \\
\hline$v A C$ & Binary variable to select V-mode charging. \\
\hline$v A c N o d e$ & Binary. It determines whether there is an ESS in the node (1) or \\
\hline$v A D$ & Binary variable to select V-mode discharging. \\
\hline$v B C$ & Binary variable to select P-mode charging. \\
\hline$v B D$ & Binary variable to select P-mode discharging. \\
\hline$v C C$ & Binary variable to check if the ESS is full. \\
\hline$v C D$ & Binary variable to check if the ESS is empty. \\
\hline$v C u t$ & Variable to prevent reverse current from appearing in substations \\
\hline$v D C$ & Binary variable to activate charging. \\
\hline$v D C$ & Binary variable to activate discharging. \\
\hline$v \quad t$ & Sampling time. \\
\hline$v E a c$ & Energy in the ESS in a given instant. \\
\hline$v P c h a r g e$ & Charging power rate. \\
\hline$v P d i s$ & Discharging power rate. \\
\hline$v P r h e o s t$ & Power sent to rheostat in a train in a given instant. \\
\hline$v T$ & Binary variable to detect rheostat braking conditions. \\
\hline$v V i$ & Voltage in the node i. \\
\hline
\end{tabular}

\section{Introduction}

Electric railway systems are inextricably energy-efficient systems. In addition to the high efficiency ratios of trains, these systems add the possibility of recovering energy from regenerative train braking, allowing it to be used again by other trains. This feature may yield important energy consumption reduction figures [1].

However, regenerative braking does not perform well in DC-electrified systems under certain conditions. In most cases, substations supplying energy to the railway network do not allow energy to flow back to the utility grid. Consequently, if no trains are demanding power during a regenerative braking, there is a power surplus that must be sent to dedicated resistors (rheostats) and hence wasted. This situation is more likely to take place in low traffic-density scenarios and may be responsible for a reduction in network energy efficiency.

There are several techniques to mitigate this effect, hence increasing system energy efficiency. Takagi [2] has analyzed the effect of reversible substations, Energy Storage Systems (ESSs) and superconducting cables. Barrero et al. [3] has researched on the effect of ESSs on a DC-electrified line with an accurate model of ESS controller. Konishi et al. [4] has performed a state-of-the-art of 
ESSs and applied them to actual cases both in energy-saving and substation mode.

In the optimization field, [5] has analysed the optimum features of a ESS in the planning stage [6] has developed a simplified model of $2 \times 25 \mathrm{kV}$ high speed railway supply systems which makes it possible to find optimal places for connections to the utility network.

However, little research has been conducted on finding a formal optimum of ESS deployment in a DC-electrified line from an energy point of view.

This paper presents a mathematical model of the electrical load flow [7] in a DC-electrified mass rapid transport system incorporating ESSs. A linearization is performed in order to get a fast approximate model of the system. This optimization model is then applied to a case-study, proving that it is possible to optimise the deployment of ESSs in the system.

\section{System modelling}

Figure 1 shows an electrical representation of the system. Four kinds of qualitatively different nodes may be observed in a DC-electrified line with ESSs:

- Slack bus: it represents the utility network connection. It is a three-phase $\mathrm{AC}$ bus which is considered to have infinite short-circuit power (infinite network).

- Substation - ESS nodes: in this approach, ESSs are only allowed to be placed in substations, i.e. in the DC bus after the transformer plus rectifier AC-DC converter.

- Train nodes: these nodes represent the non-fixed part of the system. Train positions evolve as a function of their speeds and line timetable.

- Power transferring nodes: the rest of nodes are defined in order to represent the fixed part of infrastructure (e.g. those points where the feeder line is connected to substations, etc.).

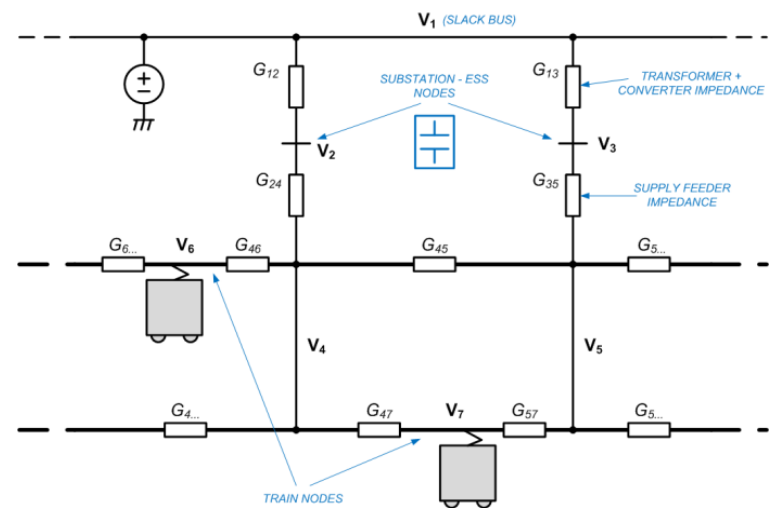

Figure 1: $\quad$ Electrical representation of the system. 
In the following paragraphs, the different modelling approaches for each component of the system are described.

\subsection{Substation - ESS nodes}

Conductances G12 and G13 in Figure 2 represent the model of the AC-DC converter that has been used. Since this study is focused on the DC side of the system, a simple model of the converter has been used. It consists of an impedance representing both losses in substations and the reactive voltage drop related to current overlapping in rectifier diodes (see [8] for further details). It must be remarked that this model simplifies dramatically the mathematical complexity of the analysis without an important loss of accuracy (for converter currents far enough from fault currents).

Then, after this impedance, an ESS may be connected (or not). A widely utilised control strategy for ESSs in railway systems is that shown in Figure 2 [4]. On the one hand, when the voltage in the feeder line rises up, which corresponds to a situation in which there is an energy surplus in the system, the ESS is commanded to start charging by trying to keep the sensed voltage in a certain level. If the power required to do that is greater than the maximum allowed power, this voltage loop is open and the ESS simply charges at its maximum allowed power rate.

On the other hand, when the voltage in the feeder line drops below a certain level, the ESS is commanded to discharge the energy it has previously stored (if

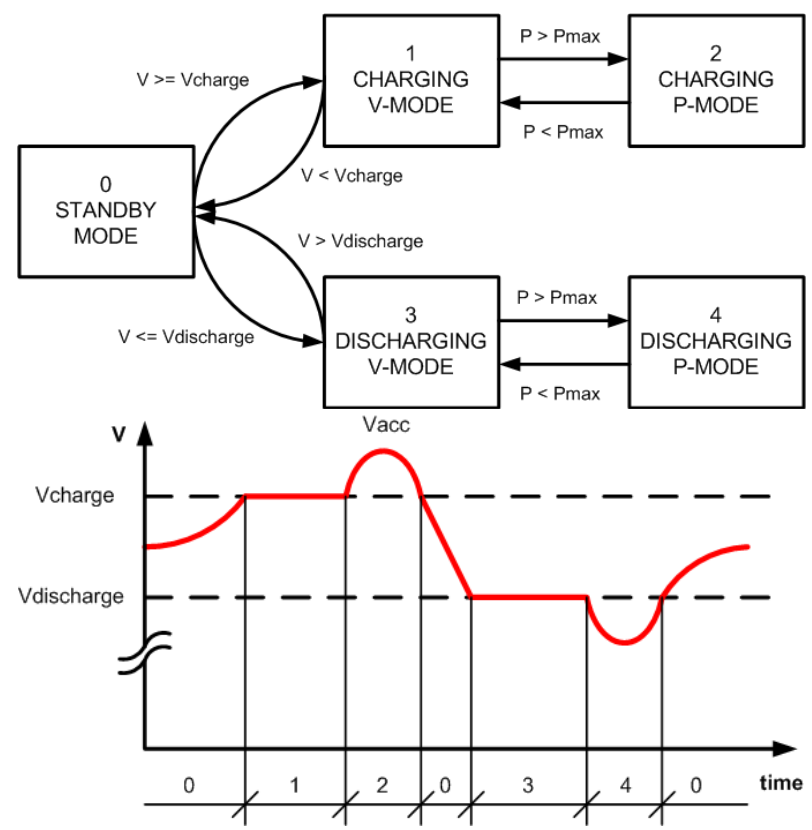

Figure 2: $\quad$ ESS control strategy and behaviour. 
available). The behaviour in this situation is analogous to that in the charge process.

ESSs have several operating modes, being energy-saving and substation (i.e., to keep the voltage high in points far away from substations where important voltage drops take place) modes the most common. In all cases, the selection of the voltage thresholds for charging and discharging is a matter of importance in these devices, since each operation mode should require specific levels for these parameters. Since the scope of ESSs in this study is to improve system energy efficiency, the discharging threshold has been set very close to the no-load voltage for the ESS to be as many times as possible ready to accept braking energy.

The following are the equations that represent the behaviour of substation nodes equipped with an ESS:

\subsubsection{Charge process: maximum energy checkout}

$$
\begin{gathered}
v E a c-p E \max \leq(1-v C C) \cdot p M \\
-(v E a c-p E \max ) \leq v C C \cdot p M
\end{gathered}
$$

\subsubsection{Charge process: ESS enable/disable}

$$
\begin{gathered}
v D C \leq v C C \\
v D C \leq v A c \text { Node } \\
v D C \geq v C C+v A c N o d e-1
\end{gathered}
$$

\subsubsection{Charge process: ESS mode 1 and 2 control}

$$
\begin{gathered}
v V-(p \text { V chrge }+(1-v D C) \cdot p \text { Vmax }) \leq v A C \cdot p M \\
-(v V-(p V \text { chrge }+(1-v D C) \cdot p V \max )) \leq(1-v A C) \cdot p M \\
v P \operatorname{chrge}-p P C \max \leq v B C \cdot p M \\
-(v P \operatorname{chrge}-p P \max ) \leq(1-v B C) \cdot p M \\
v P \operatorname{chrge} \geq 0 \\
v P \operatorname{chg} e \leq v A C \cdot p P C \max
\end{gathered}
$$

These equations express the charging process described in Figure 2.

\subsubsection{Discharge process}

The discharge process is expressed by the same (analogous) equations than the charge process. 


\subsubsection{ESS voltage control}

$$
\begin{gathered}
v V \geq(1-v B D) \cdot p \text { Vdis } \\
v V \leq p \operatorname{chrge}+(p V \max -p V \operatorname{chrge}) \cdot v B C \\
+(p V \max -p \operatorname{chrge}) \cdot(1-v \text { AcNode })
\end{gathered}
$$

Voltage in the node is limited to the maximum admissible power or to the charging threshold, depending on ESS state (if there is an ESS in the node).

\subsubsection{Substation cut-off checkout}

$$
\begin{gathered}
v V-p V 1 \leq v \text { Cut } \cdot p M \\
-(v V-p V 1) \leq(1-v C u t) \cdot p M
\end{gathered}
$$

Variable vCut is used to prevent reverse current from appearing at substations.

\subsubsection{Power balance (non linear)}

$$
\begin{aligned}
& \text { vPdischrge }-v \text { Pchrge } \\
& \qquad \begin{array}{l}
=p V 1 \cdot v V_{i} \cdot(1-v C u t) \cdot p G_{1, i}+v V_{i}^{2} \\
\cdot\left(p G_{i, i}+v C u t \cdot p G_{1, i}\right)+\sum_{\substack{j=2 \\
j \neq i}} v V_{i} \cdot v V_{j} \cdot p G_{i, j}
\end{array}
\end{aligned}
$$

This equation expresses the power flow in the node. The variable vCut removes the linkage to the slack node when current in substation tends to reverse.

\subsection{Train nodes}

Trains have been modelled as power loads. This is the most accurate model, regarding modern rolling stock power chain features. Power is positive in traction moments and negative during braking.

A binary variable is activated when train voltage reaches a certain voltage threshold. When this situation takes place, the braking power surplus is sent to rheostats, and hence wasted. Otherwise, rheostat power remains zero.

The following are the equations that represent the behaviour of train nodes.

\subsubsection{Rheostat power modelling}

$$
\begin{gathered}
v V-p V \max \leq v T \cdot p M \\
-(v V-p V \max ) \leq(1-v T) \cdot p M
\end{gathered}
$$




$$
\begin{gathered}
v \text { Prheost } \leq v T \cdot \text { pPgen } \\
v \text { Prheost } \geq 0 \\
v V \geq 0 \\
v V \leq p V \max
\end{gathered}
$$

\subsubsection{Power balance (non linear)}

$$
\text { pPgen }- \text { PPdem }-v \text { Prheost }=\sum_{j=1}^{N} v V_{i} \cdot v V_{j} \cdot p G_{i, j}
$$

This equation expresses the power flow in the train node.

\subsection{Power transferring nodes}

These nodes are used to define the fixed part of the infrastructure. They add a non linear power balance equation similar to that in the train case, but the power injected or consumed in the node equals 0 .

$$
0=\sum_{j=1}^{N} v V_{i} \cdot v V_{j} \cdot p G_{i, j}
$$

\subsection{Optimisation problem definition}

Once the electrical system is modelled, a set of time instants is generated in order to be able to assess how much energy may be saved by incorporating energy storage devices to the infrastructure. Trains are placed in the line at each instant following a given traffic scenario.

Then, a snapshot of system power demand and regeneration is obtained at a certain sampling time.

Since ESS behaviour may vary as a function of their state of charge, their energy variation is recorded at each instant:

$$
v E a c_{k}=v E a c_{k-1}+(v P \operatorname{chrge} \cdot p E f f-v P d i s / p E f f) \cdot v \Delta t
$$

In this approach, which is aimed at obtaining an optimum energy solution, the objective function of the optimisation problem has been defined as energy consumption at substations. In a refined approach, economical concerns could be taken into account in order to assess investments. 


\section{Linearization}

Several problems are expected with the current non-linear modelling approach. The MINLP formulation of the problem has exhibited certain convergence problems. In order to get rid of these problems, a linearization is proposed.

Power balance equations (16), (23), (24) present two types of non-linearity: first, all of them contain the addition of products of couples of real variables; second, equation (16) presents a real-binary variable product.

In order to deal with the first non-linearity, voltages in the system have been expressed as the sum of a fixed part plus an increment. Then, the following approximation has been carried out:

$$
\begin{aligned}
v V_{i} \cdot v V_{j}=\left(v V_{i}^{0}+v \Delta V_{i}\right) \cdot\left(v V_{j}^{0}+v \Delta V_{j}\right) & \\
& \approx v V_{i}^{0} \cdot v V_{i}^{0}+v V_{i}^{0} \cdot v \Delta V_{j}+v V_{j}^{0} \cdot v \Delta V_{i}
\end{aligned}
$$

The product of increments has been neglected. Under this approximation, if the operation point is set at the flat start (all voltages in the network set to the nominal no-load voltage), it may be proved that energy in substations equal energy in trains, hence resulting in a lossless model of the load flow.

The second non-linearity (real-binary product) has been solved replacing the non linear term by a real variable and adding linear restrictions which make them be equivalent [9].

\section{Case study}

In this study, ESSs have been placed in a realistic line. The main characteristics of this line are the following:

- Nine passenger stations distributed along $5 \mathrm{~km}$.

- Three electrical substations equipped with twelve-pulse rectifiers. They are connected to $15 \mathrm{kV}$ utility company's buses and they provide $1.5 \mathrm{kV}$ no-load voltage.

Trains are operated in a double track with rigid overhead conductor. There are no isolators to electrically segment the line and conductors are paralleled in substations. Both rails carry the return current back to substations, where return lines are earthed.

The rolling stock material used in the simulations has the following features:

- Maximum traction power: $1.5 \mathrm{MW}$.

- Maximum braking power (in regenerative braking cases): $1.5 \mathrm{MW}$.

- Consumption of auxiliary equipment: $200 \mathrm{kWh} / \mathrm{h}$.

The following practical conventions have been used:

- Flat out train speed profiles have been simulated.

- Trains have been launched from both sides of the line at a fixed 7-minute frequency.

- The no-load voltage has been set to its nominal value $(1.5 \mathrm{kV})$.

- ESS maximum energy storage has been set to $12 \mathrm{kWh}$. The maximum admissible power has been set to $700 \mathrm{~kW}$. 


\section{Results}

\subsection{ESS deployment}

The presented MIP model has been implemented in GAMS language and run in a PC equipped with an 8 -core $3.4 \mathrm{GHz}$ processor and 8GB RAM memory, running Microsoft Windows 7. The MIP problem has been solved using CPLEX 12.3.0.0. An accurate non linear model implemented in MATLAB [10] has been used as a reference for assessing linear-model error.

In order to check that the optimal solution with this approximate model matches the optimal solution using an accurate non linear model, two scenarios have been simulated: In the first scenario, the maximum number of ESSs in the system is limited to 1 , while in the second one this restriction is relaxed and hence the maximum number of ESSs allowed is 3 .

The linear model obtains the minimum energy consumption $(1.31 \mathrm{MWh} / \mathrm{h}$ vs. $1.32 \mathrm{MWh}$ with the accurate model) when an ESS is placed at substation 3 . Figure 3 shows energy consumption results obtained with the accurate model when only one ESS is allowed. It may be observed that the minimum energy consumption takes place when the ESS is placed at substation 3, as predicted by the linear model.

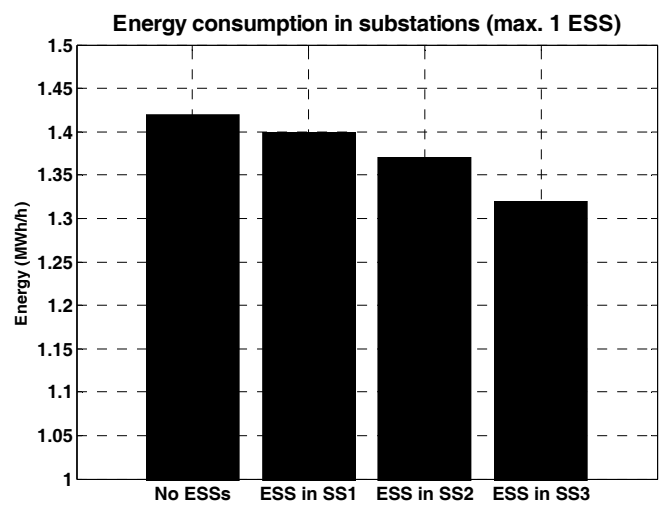

Figure 3: Accurate energy consumption in substations (only 1 ESS allowed).

When the maximum number of ESSs restriction is relaxed, the minimum energy consumption $(1.29 \mathrm{MWh} / \mathrm{h})$ is obviously obtained when an ESS is placed at every substation (i.e. at every possible placement), resulting in zero rheostat losses. This is in line with results obtained with the non linear model, confirming that the linear model evolves properly to the global optimum.

Computation times measured are $20.03 \mathrm{~s}$ in the first scenario and $34.63 \mathrm{~s}$ in the second one. 


\subsection{Error evaluation}

In this section, the error due to the linearization of node load flow equations is assessed. Two types of errors have been taken into account: voltage and power error. The case with an ESS placed at substation 3 has been used for error evaluation.

It may be observed in Figure 4 that percent voltage error remains within a $\pm 0.25 \%$ interval for all nodes and time instants. Voltage error is greater as voltages are further from the no-load voltage.
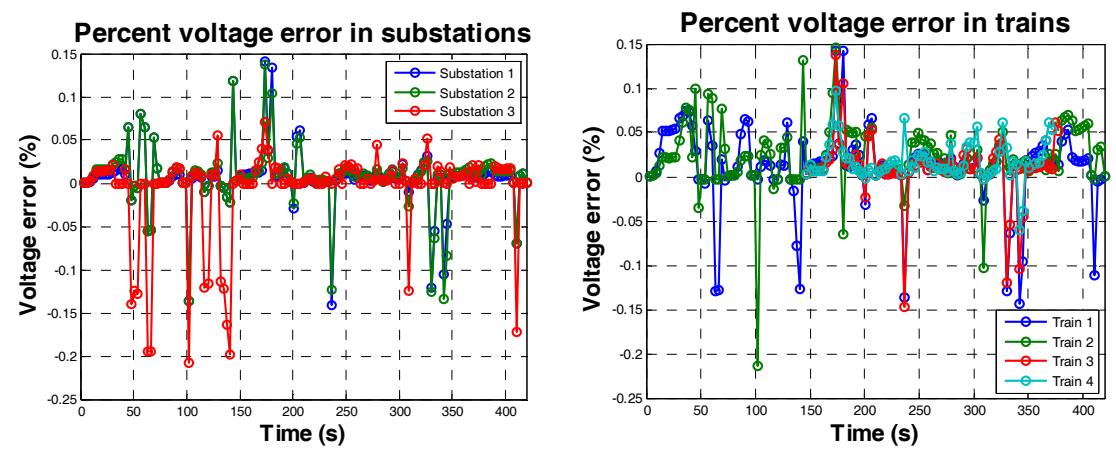

Figure 4: $\quad$ Percent voltage error in substation and train nodes.

Figure 5 shows power error during charge and discharge of the ESS. Absolute errors are compared with total power in the ESS. It may be observed that power in ESSs predicted by the linear model is fairly close to the accurate result. In those instants where ESS works in maximum power mode both power are forced to be the maximum value $(700 \mathrm{~kW})$, and hence error becomes zero. The main reason for these accurate results is the system present low losses figures, which is in turn due to the rigid catenary overhead conductor and to the low distance between electrical substations.

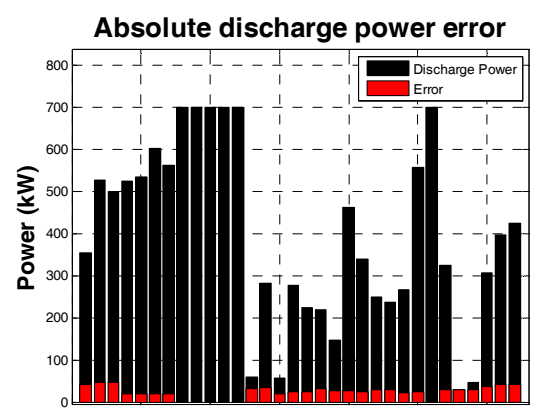

Instances of ESS discharge

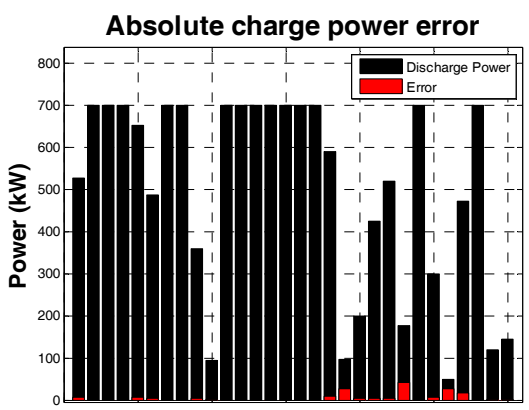

Instances of ESS charge

Figure 5: Discharge and charge power error vs. total power. 
Figure 6 shows energy evolution in the ESS in substation 3. Results in the linear model are close to accurate results. However, since discharges in the linear approach are always lower than actual discharges, an offset is observed when several discharges take place. This may be corrected by introducing this offset in the discharge model.

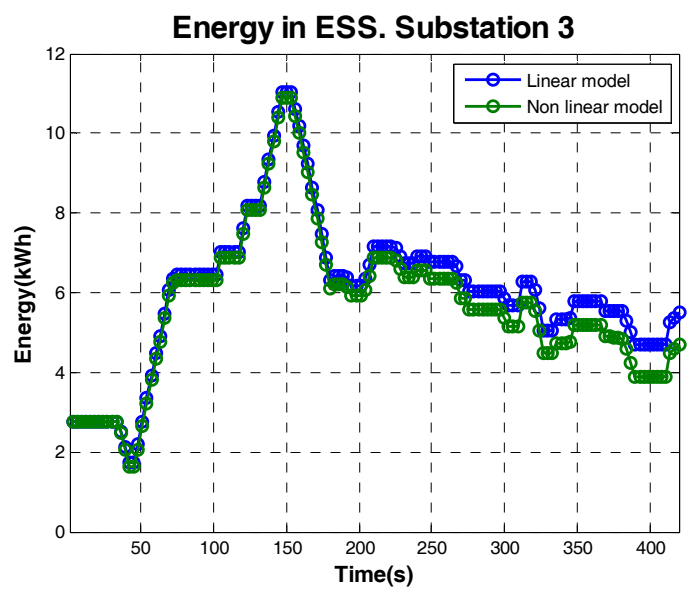

Figure 6: Energy evolution in the ESS.

An energy swing of around $8.5 \mathrm{kWh}$ is observed in the ESS. This result may be regarded as good value for dimensioning ESS available energy swing for this system, and for the case that only one ESS is placed in the line.

\section{Conclusions}

The linear version of the load flow in a DC-electrified railway line has yielded results which are in line with results from exhaustive analysis, hence assessing optimal ESS deployment.

Voltage and power approximate results have been compared with those of an accurate non linear model, showing that this model represents a fairly good approximation for systems with low losses.

The linear model presents advantages in the application to large scale systems. Some ESS characteristics that have been treated as fixed parameters in this study such as charge and discharge thresholds or maximum storage energy may be formulated as optimization variables, hence obtaining both optimum ESS layout and features.

In the future, this linear model is to be extended to a full railway system analysing several traffic scenarios with uncertainties. These scenarios are intended to cluster the different traffic conditions that take place in the line. Composing them appropriately, representative yearly savings may be obtained. 
In addition, energy consumption reduction with regard to the case without ESSs will be converted into economic saving and compared with investment and maintenance costs in the objective function.

\section{References}

[1] W. Gunselmann, "Technologies for increased energy efficiency in railway systems," in 2005 IEEE 11th European Conference on Power Electronics and Applications, 2005.

[2] R. Takagi, "Energy Saving Techniques for the Power Feeding Network of Electric Railways," IEEJ Transactions on Electrical and Electronic Engineering, vol. 5, pp. 312-316, 05, 2010.

[3] R. Barrero, X. Tackoen and J. Van Mierlo, "Improving energy efficiency in public transport: Stationary supercapacitor based energy storage systems for a metro network," in 2008 IEEE Vehicle Power and Propulsion Conference (VPPC), 2008.

[4] T. Konishi, H. Morimoto, T. Aihara and M. Tsutakawa, "Fixed Energy Storage Technology Applied for DC Electrified Railway," IEEJ Transactions on Electrical and Electronic Engineering, vol. 5, pp. 270-277, 05, 2010.

[5] L. Battistelli, F. Ciccarelli, D. Lauria and D. Proto, "Optimal design of DC electrified railway stationary storage system," in 2009 International Conference on Clean Electrical Power (ICCEP), 2009.

[6] E. Pilo, L. Rouco, A. Fernandez and L. Abrahamsson, "A monovoltage equivalent model of bi-voltage autotransformer-based electrical systems in railways," IEEE Transactions on Power Delivery, vol. 27, pp. 699-708, 04/01, 2012.

[7] J. Arrillaga and N. R. Watson, Computer Modelling of Electrical Power Systems. 2001.

[8] Y. S. Tzeng and R. N. Wu, "A detailed R-L fed bridge converter model for power flow studies in industrial AC/DC power systems," IEEE Transactions on Industrial Electronics, vol. 42, 1995.

[9] S. Walukiewicz, Integer Programming. Kluwer Academic Publishers, 1991.

[10] A. J. López López, R. R. Pecharromán, E. Pilo, A. P. Cucala and A. Fernández-Cardador, "Analysis of energy-saving strategies in railway power supply systems," 9th World Congress on Railway Research - WCRR 2011, 22-26 May, 2011. Lille, France. 